\title{
comportement du remblai expérimental B à Cubzac-les-Ponts
}

\author{
par \\ J.P. Magnan \\ Laboratoire Central des Ponts et Chaussées \\ C. Mieussens \\ Laboratoire Régional des Ponts et Chaussées de Toulouse \\ et \\ D. Queyroi \\ Laboratoire Régional des Ponts et Chaussées de Bordeaux
}

\section{Introduction}

En 1972 les Laboratoires des Ponts et Chaussées ont entrepris une campagne d'étude de remblais sur sols mous sur un site expérimental situé dans la commune de Cubzac-les-Ponts au nord de Bordeaux, en bordure de la Dordogne. Trois remblais expérimentaux étaient prévus sur ce site :

- le remblai A (juin 1974) devait être construit jusqu'à la rupture, le but de l'expérience étant l'étude du comportement du sol juste avant la rupture et de la forme du glissement. Les conclusions de cette étude ont été présentées par Vogien (1975) et Blondeau et al. (1977). La hauteur du remblai $A$ lors de la rupture $(4,50 \mathrm{~m})$ a permis de donner aux remblais suivants une hauteur correspondant exactement au coefficient de sécurité désiré ;

- le remblai B (octobre 1975) a été édifié avec un coefficient de sécurité de 1,5, qui est la valeur recommandée en France pour les remblais routiers courants. Compte-tenu des enseignements du remblai $A$, sa hauteur a été fixée à $2,30 \mathrm{~m}$. Ce remblai, destiné à l'étude de la consolidation du sol de fondation sous le remblai et dans son voisinage, est l'objet de constatations régulières depuis sa construction. II sert d'exemple pour la comparaison des méthodes de calcul pour remblais sur sols compressibles ;

- le remblai $C$, dont la construction est prévue pour juin 1978, sera édifié avec un coefficient de sécurité plus faible $(F=1,2)$ pour étudier le comportement du sol de fondation dans des conditions plus proches de la rupture.

\section{Le site expérimental de Cubzac-les-Ponts}

\subsection{Localisation}

Le site expérimental est situé dans la commune de Cubzac-les-Ponts, en bordure de l'Autoroute A10 (" L'Aquitaine 11$)$, sur la rive nord de la Dordogne (fig. 1). La cote N.G.F. au niveau du sol est de $+2 \mathrm{~m}$.

A cet endroit les alluvions compressibles de la vallée de la Dordogne (fig. 2) comportent :

- une mince couche de terre végétale d'épaisseur approximative $0,3 \mathrm{~m}$;

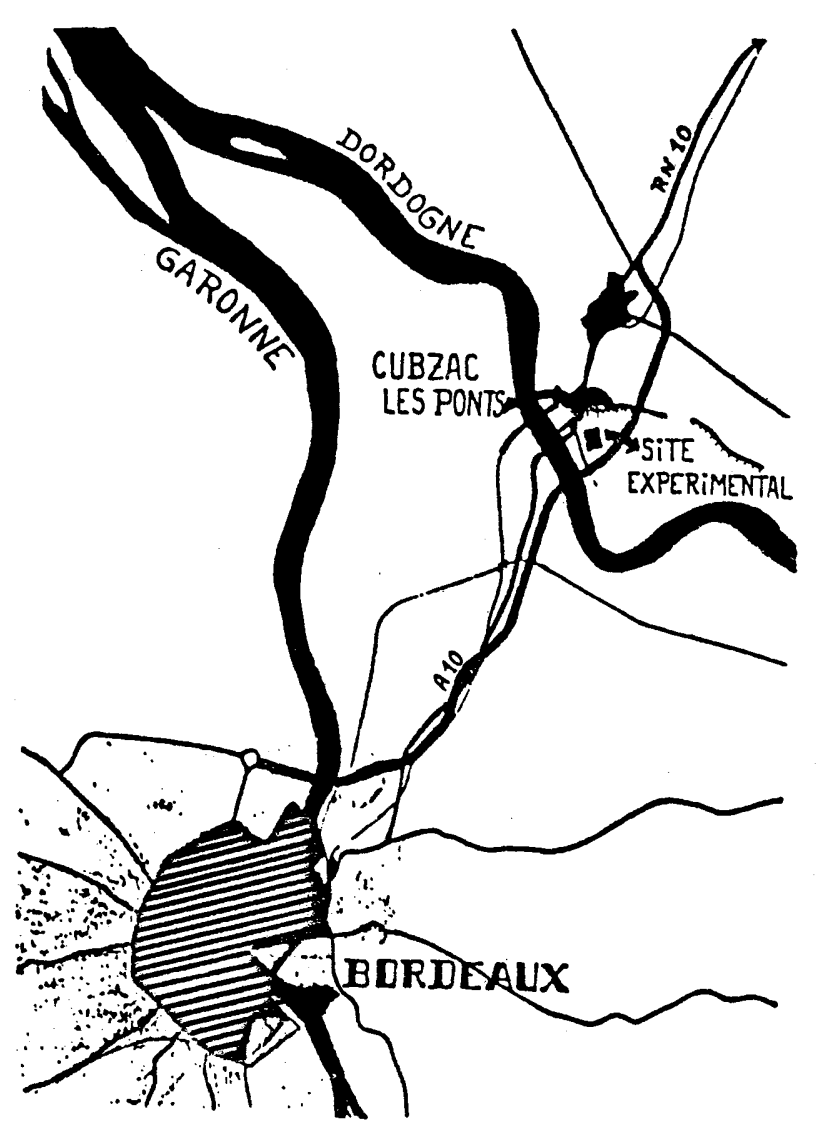

Fig. 1 Localisation du site expérimental de Cubzacles-Ponts 


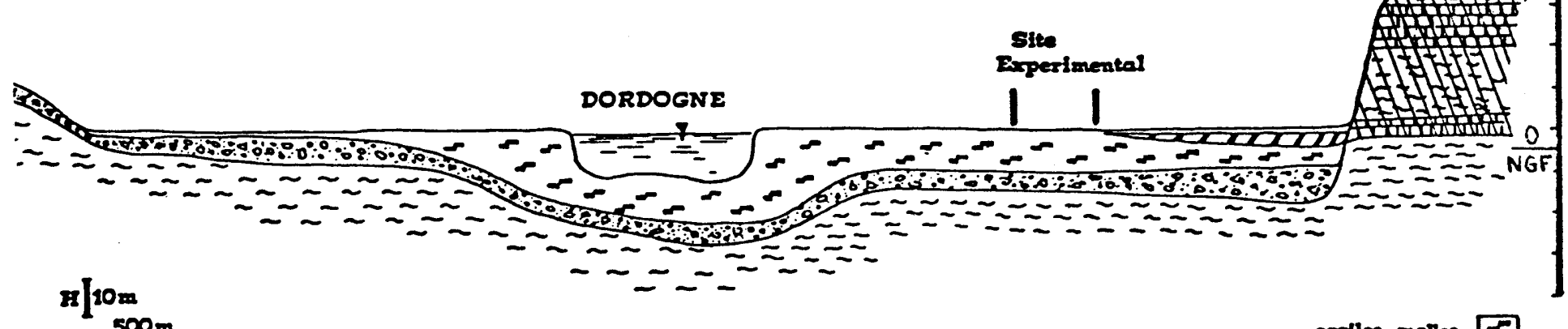

500

2

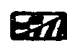

R्य

$8 \mathrm{R}$

저

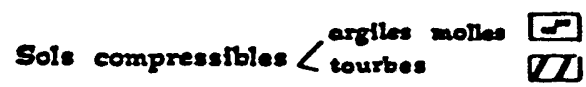

Mlupions arcienne:

Stampien

Roche marneuse or calcalre

Fig. 2 Coupe géotechnique transversale de la vallée de la Dordogne à la hauteur de Cubzac-les-Ponts

- une couche d'argile limoneuse d'épaisseur inférieure à $2 \mathrm{~m}$ formant une croûte de surface surconsolidée et altérée par dessiccation. (Le niveau de la nappe phréatique varie du terrain naturel en hiver à $-1,5 \mathrm{~m}$ en été. Lors de la construction du remblai $B$ en octobre 1975 la nappe était située à $-1,5 \mathrm{~m})$;

- une couche d'argile molle grise plus ou moins organique légèrement surconsolidée et dont l'épaisseur atteint $8 \mathrm{~m}$ (sous le remblai B on peut diviser cette couche en deux parties: une couche plus organique de $-2 \mathrm{~m}$ a $-6 \mathrm{~m}$ et une couche moins organique, de $-6 m$ a $-8,5 m$ ).

Le substratum est constitué d'une couche de graves située vers 9 a $10 \mathrm{~m}$ de profondeur, d'épaisseur moyenne $5 \mathrm{~m}$, reposant sur des roches marneuses ou calcaires.

L'influence de la marée sur les pressions interstitielles du site est nulle.

\subsection{Caractéristiques géotechniques de la vase de Cubzac}

Le site expérimental a fait l'objet de plusieurs campagnes de reconnaissance géotechnique détaillée, lors de l'étude des remblais de l'autoroute voisine, lors de l'étude préliminaire au choix du site, lors de l'étude du remblai $A$, lors de l'étude du remblai $B$ et lors de celle du remblai $C$.
Les trois dernières reconnaissances ont montré, contrairement aux conclusions de la campagne initiale, réalisée avec une maille de sondage plus lâche, que les caractéristiques géotechniques du sol varient de près de $20 \%$ à l'intérieur du site, dont les dimensions sont pourtant restreintes $(170 \mathrm{~m} \times 225 \mathrm{~m})$.

La figure 3 présente une synthèse des propriétés moyennes du sol à l'emplacement du remblai B (Blondeau et al. (1977) ont donné un diagramme analogue pour le remblai A).

Sous la croûte de surface, la vase de Cubzac est légèrement surconsolidée (rapport de surconsolidation voisin de $1,2)$, fortement compressible $(\mathrm{Cc} /(1+\mathrm{eo})$ voisin de 0,4$)$, très organique (teneur en matières organiques comprise entre 5 et $25 \%$ ) et très plastique (I $P$ compris entre 50 et 100 - et $W_{L}$ entre 100 et 200 ) et sa teneur en eau naturelle est voisine de la limite de liquidité. La cohésion non drainée Cuest faible (de 15 à $20 \mathrm{kPa}$ entre $2 \mathrm{~m}$ et $7 \mathrm{~m}$ de profondeur).

\subsection{Disposition des remblais}

La figure 4 présente la disposition des remblais dans le site expérimental. Le remblai $B$ a été construit avec le matériau du remblai $A$, conduit à la rupture l'année précédente. Le remblai C n'était pas encore édifié lors de la préparation de cet article.

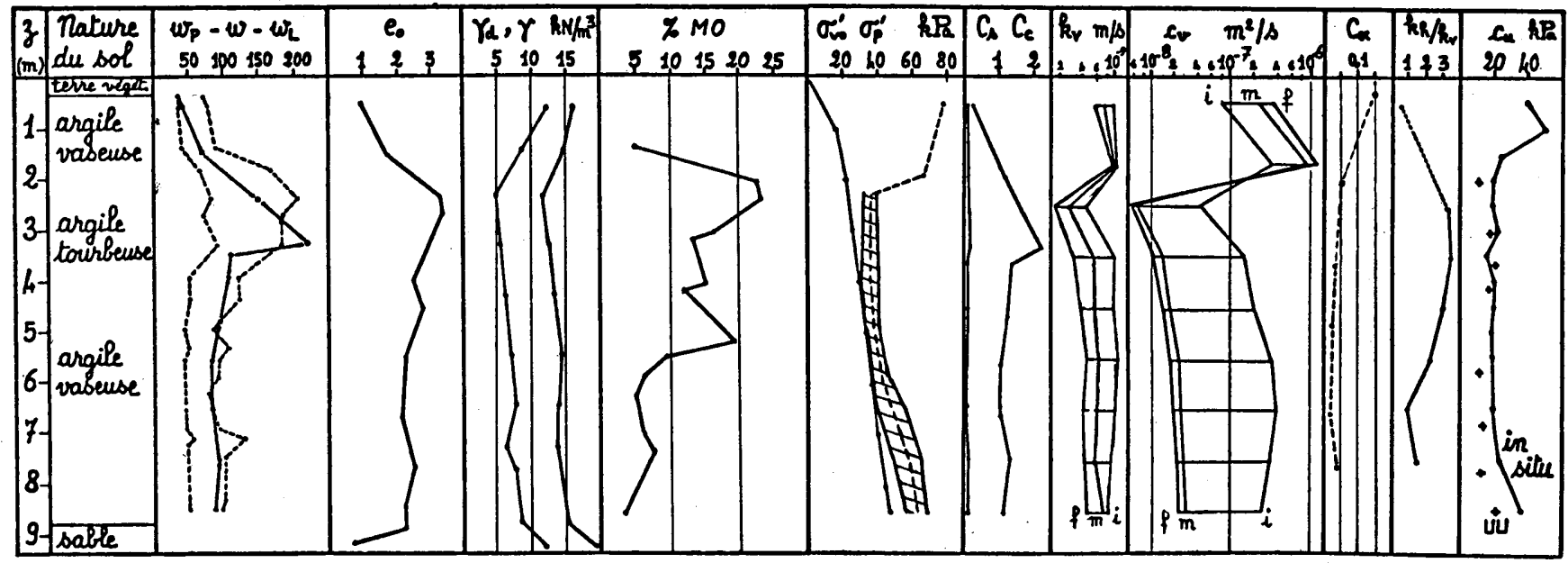

Fig. 3 Caractéristiques géotechniques du sol de fondation du remblai B (Cubzac-les-Ponts)

Pour $\mathrm{k}_{\mathrm{v}}$ et $\mathrm{c}_{\mathrm{v}}$ les indices $\mathrm{i}, \mathrm{m}, \mathrm{f}$ correspondent aux courbes obtenues sous $\sigma_{\mathrm{vo}}^{\prime}, \sigma_{\mathrm{vo}}^{\prime}+\frac{\Delta \sigma}{2}$ et $\sigma_{\mathrm{f}}^{\prime}=\sigma_{\mathrm{vo}}^{\prime}+\Delta \sigma$ 


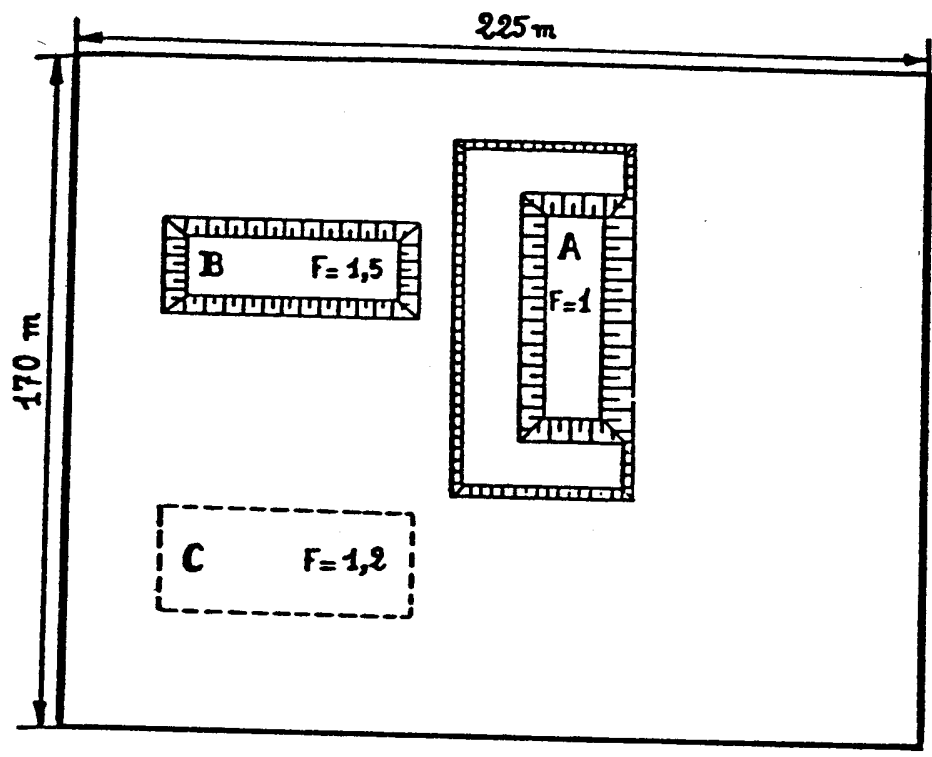

Fig. 4 Implantation des remblais du site expérimental de Cubzac-les-Ponts

\section{Le remblai B}

\subsection{Dimensions}

La figure 5 présente les dimensions retenues pour assurer au remblai $B$ un coefficient de sécurité de 1,5. La longueur du remblai a été choisie pour que la zone centrale se déforme dans des conditions pratiquement bidimensionnelles (déformations planes), comme dans un ouvrage réel. La largeur est environ le double de l'épaisseur de la couche compressible.

\subsection{Plan d'instrumentation}

L'instrumentation du sol de fondation du remblai B a été terminée deux mois avant la construction du remblai pour éviter toute influence de la mise en place des appareils sur les mesures.
Les appareils de mesure sont au nombre de 108, soit : - 45 piézomètres à contre-pression hydraulique (type
LPC)

14 piézomètres à contre-pression pneumatique (type
LPC)

- 3 piézomètres électriques à jauge (type LRPC Bordeaux)

- 20 tassometres hydrauliques de profondeur (type LPC)

- 7 tassomètres hydrauliques de surface (type LPC)

- 3 capteurs de déformation différentielle horizontale (type LPC ; cf. Blondeau et al., 1977)

16 capteurs électriques de contraintes totales (type LRPC, Bordeaux), plus 3 tubes inclinométriques et 114 jalons disposés à la surface du sol autour du remblai. La plupart de ces appareils de mesure sont installés dans le profil transversal central (profil K) du remblai ; mais on a aussi équipé deux profils transversaux de contrôle distants de $10 \mathrm{~m}$ du profil $K$ (profil I et $M$ ) ainsi que lo profil $O$ (axe longitudinal du remblai).

Les figures 6,7 et 8 indiquent la disposition des appareils de mesure dans le profil $K$.

\subsection{Construction du remblai}

Le matériau utilisé pour le remblai B provient en totalité du remblai $A$. II s'agit essentiellement de graviers et de gros sable :

$\%$ passant à $0,2 \mathrm{~mm}: 2 \%$

$\%$ passant à $20 \mathrm{~mm}: 75 \%$

L'absence presque totale de fines entraîne que ce matériau ne présente aucune cohésion $\left(c^{\prime}=0\right)$. Son angle de frottement interne vaut $\varnothing=35^{\circ}$.

Les mesures de densité en place exécutées pendant la construction du remblai $\mathrm{B}$ ont donné comme poids volumique moyen du matériau de remblai :

$$
\gamma=21 \mathrm{kN} / \mathrm{m}^{3} \text {. }
$$

Ce poids volumique est très élevé.

Le remblai a été construit assez rapidement en 3 couches et à une vitesse assez régulière à part l'interruption de la fin de semaine (samedi-dimanche) entre la première couche $(0,70 \mathrm{~m})$ et les trois couches suivantes $(1,20 \mathrm{~m}-1,90 \mathrm{~m}$ et $2,30 \mathrm{~m}$ ). En fin de construction, le remblaí a atteint une hauteur de $2,3 \mathrm{~m}$ au-dessus du terrain naţurel, que l'on peut considérer comme horizontal.

La figure 9 présente le calendrier de construction du remblai.

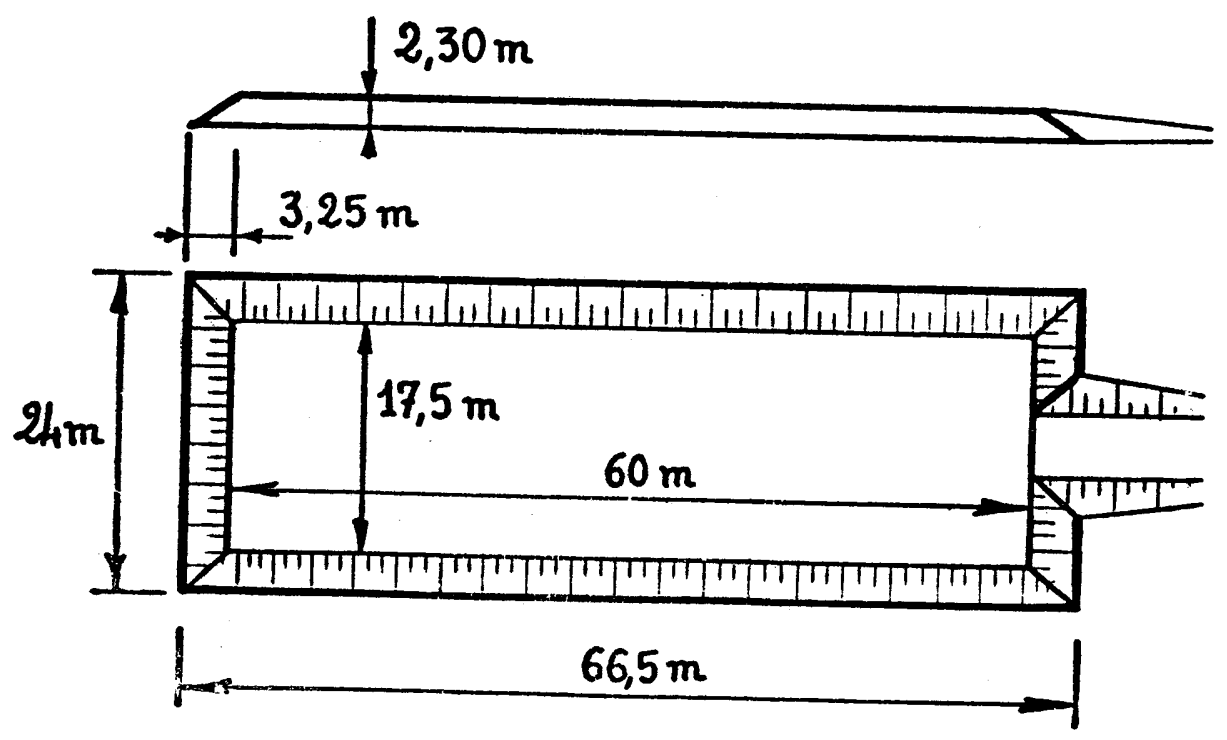

Fig. 5 Dimensions du remblai B 

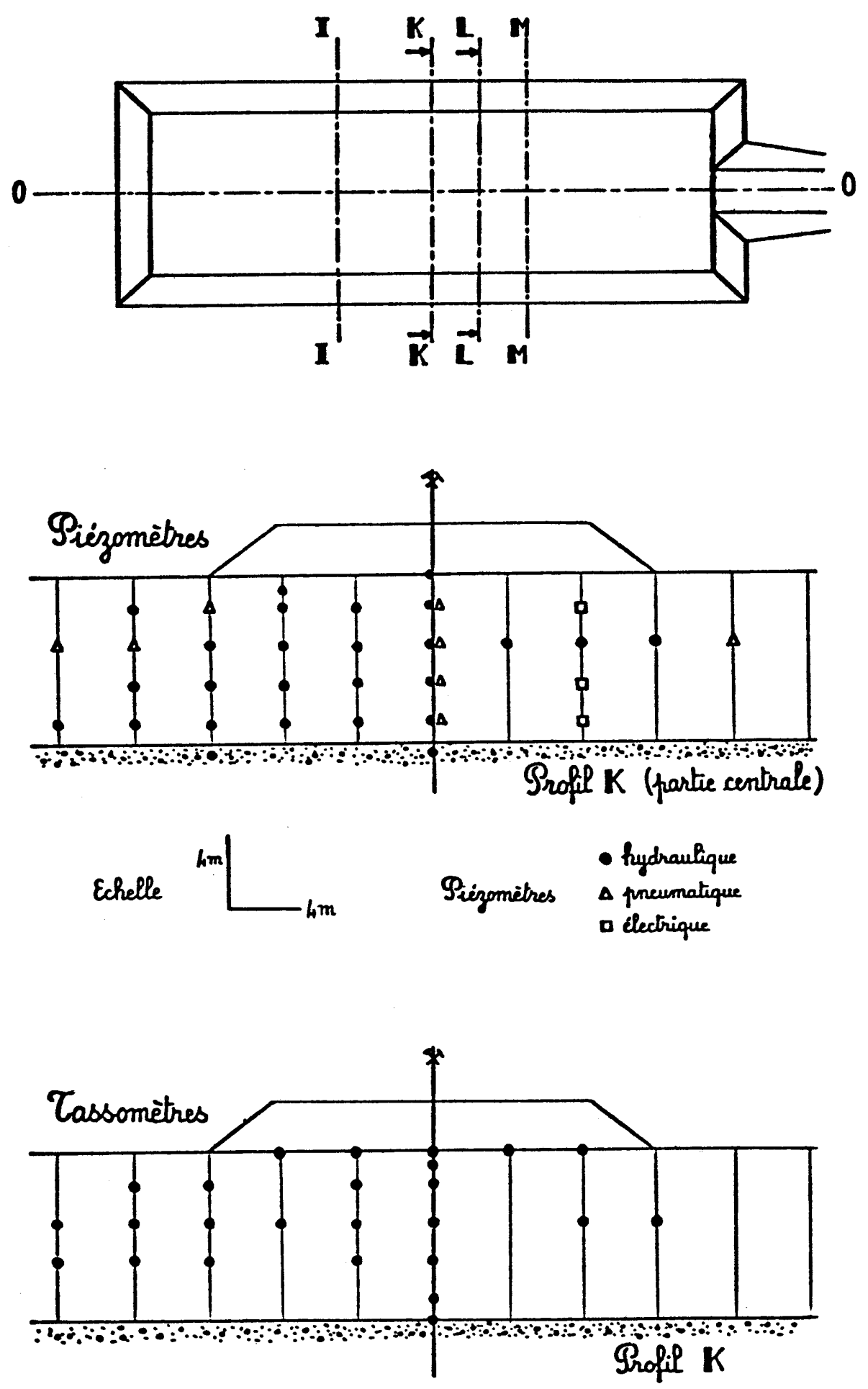

Fig. 6 Instrumentation du remblai B : piézomètres et tassomètres 
Inclinometres et c.d.d.h.
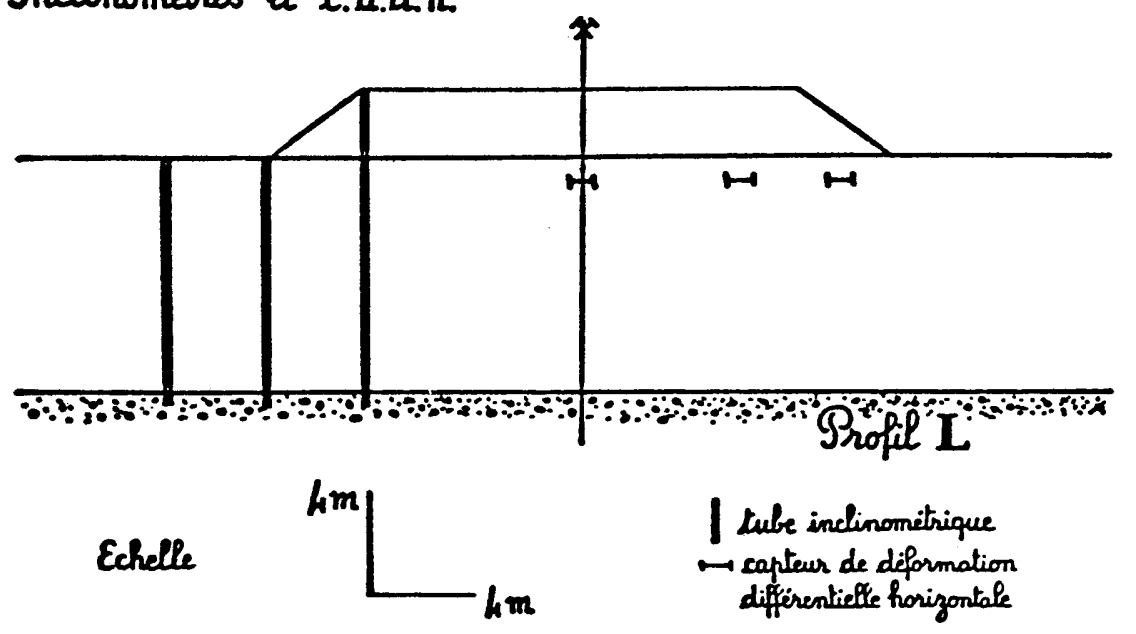

Capteurs de contraintes totales

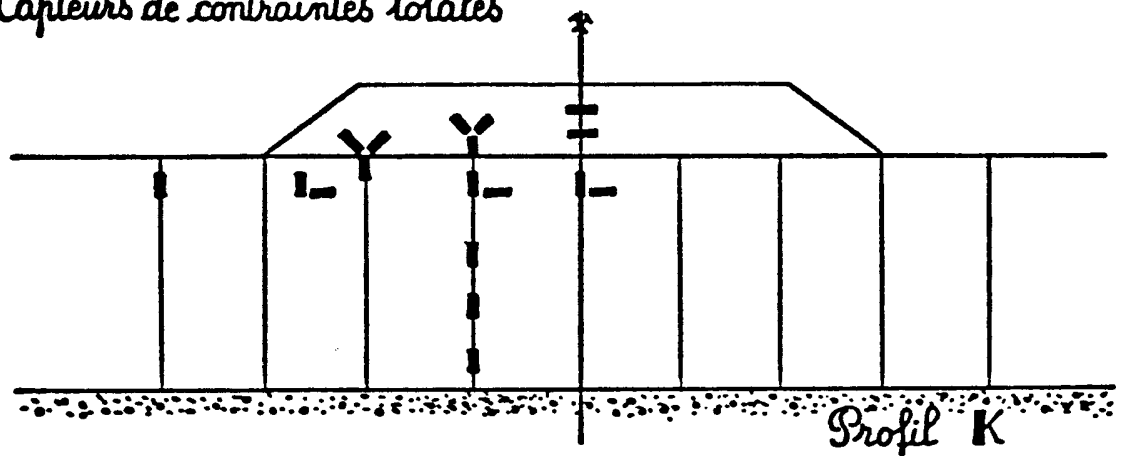

Fig. 7 Instrumentation du remblai B : inclinomètres et capteurs de déformation différentielle horizontale et de contraintes totales

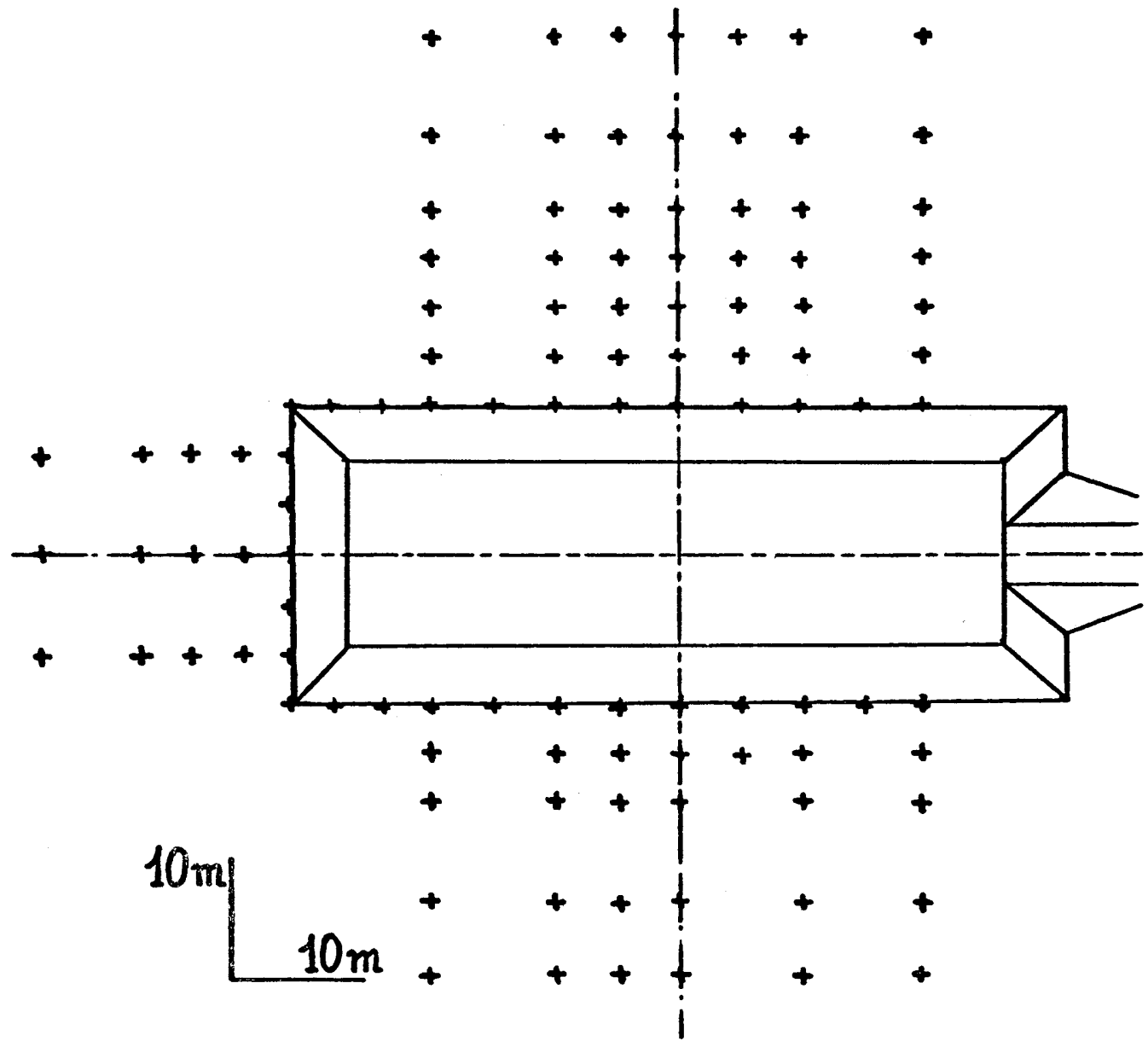

Fig. 8 Instrumentation du remblai B : jalons (repères topographiques) 


\begin{tabular}{|c|c|c|}
\hline Couche & $\begin{array}{c}\text { Hauteur } \\
\text { du } \\
\text { remblai }\end{array}$ & \multicolumn{1}{|c|}{ Date $/$ Heure } \\
\hline \multirow{2}{*}{1} & $0,70 \mathrm{~m}$ & $\begin{array}{l}9 . \times .75 \mathrm{a} \\
10 . \times .75 / 11 \mathrm{~h} 30\end{array}$ \\
\hline \multirow{2}{*}{2} & $1,20 \mathrm{~m}$ & $13 . \times .75 / 14 \mathrm{~h} \mathrm{00}$ \\
\cline { 2 - 3 } & $1,60 \mathrm{~m}$ & $14 . \times .75 / 11 \mathrm{~h} \mathrm{30}$ \\
\cline { 2 - 3 } & $1,90 \mathrm{~m}$ & $14 . \times .75 / 17 \mathrm{~h} \mathrm{00}$ \\
\hline 3 & $2,30 \mathrm{~m}$ & $15 . \times .75 / 12 \mathrm{~h} \mathrm{00}$ \\
\hline
\end{tabular}

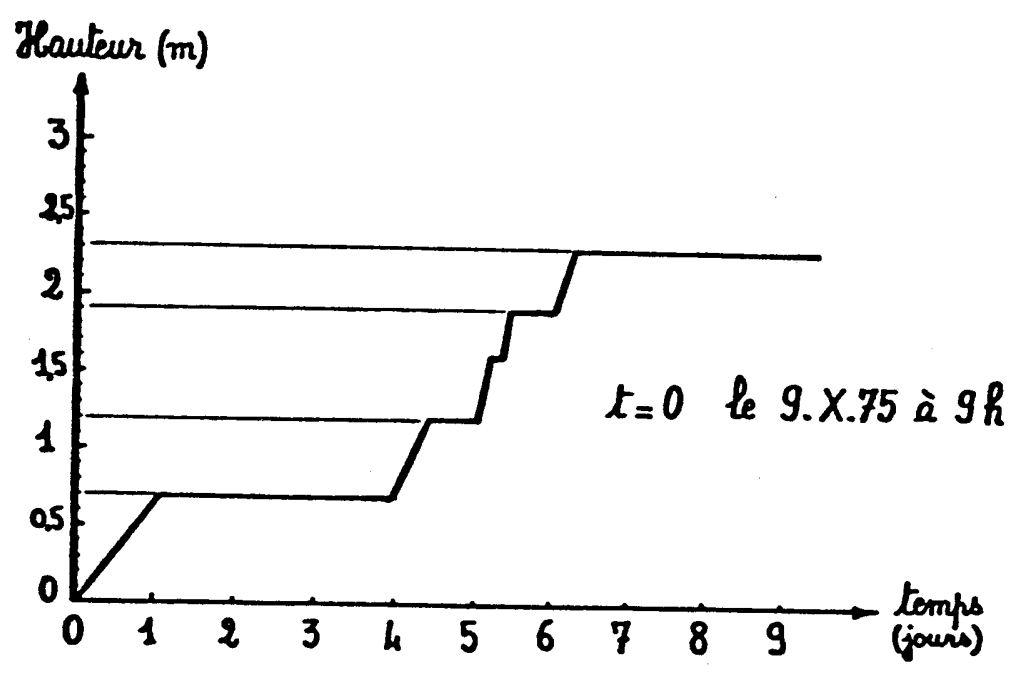

Fig. 9 Calendrier de la construction du remblai $B$

\section{Comportement du remblai au cours du temps}

L'évolution des déformations du sol et du remblai, des pressions interstitielles et des contraintes totales a été suivie régulièrement depuis le début de la construction.

\section{1 Évolution des tassements}

La figure 10 présente l'évolution des tassements du sol de fondation dans le profil transversal $K$. On note le tassement sensible du pied de talus du remblai depuis le mois de mars 1977, c'est-à-dire seize mois après la construction du remblai, et le gonflement simultané du sol au-delà du bourrelet initial. Ce phénomène peut avoir son origine dans la consolidation des couches compressibles profondes.

La figure 11 présente l'évolution au cours du temps du tassement du sol à différents niveaux dans l'axe du remblai (tassometres placés à $0 \mathrm{~m},-1 \mathrm{~m},-2 \mathrm{~m},-4 \mathrm{~m}$, et $-6 \mathrm{~m}$ par rapport au niveau du terrain naturel). Des difficultés de mesure (désaturation des tassomètres hydrauliques entre les mesures, du fait peut-être de la porosité des rilsans aux gaz présents dans le sol (méthane)), rendent imprécises les mesures des tassements à $6 \mathrm{~m}$ de profondeur $( \pm 3 \mathrm{~cm})$ et inexploitables celles qui ont été réalisées à $8 \mathrm{~m}$.

L'analyse des tassements par couche de $1 \mathrm{~m}$ de sol (entre les tassometres) montre (fig. 12) que le tassement a été très rapide dans les couches de surface, qui ont pratiquement cessé de tasser à l'heure actuelle, alors que la consolidation est en cours pour le centre de la couche compressible.

\subsection{Déplacements latéraux du sol de fondation}

Les mesures inclinométriques réalisées dans les tubes implantés en crête, en pied et au-delà du pied du remblai montrent que les déplacements horizontaux, de l'ordre de $12 \mathrm{~cm}$ sous le talus, semblent s'être stabilisés depuis le mois de Juin 1977 (vingt mois après la construction). Entre Avril et Juin 1977 le tube inclinométrique implanté en pied de talus s'est pourtant déplacé de plus de $4 \mathrm{~cm}$ et l'on a 


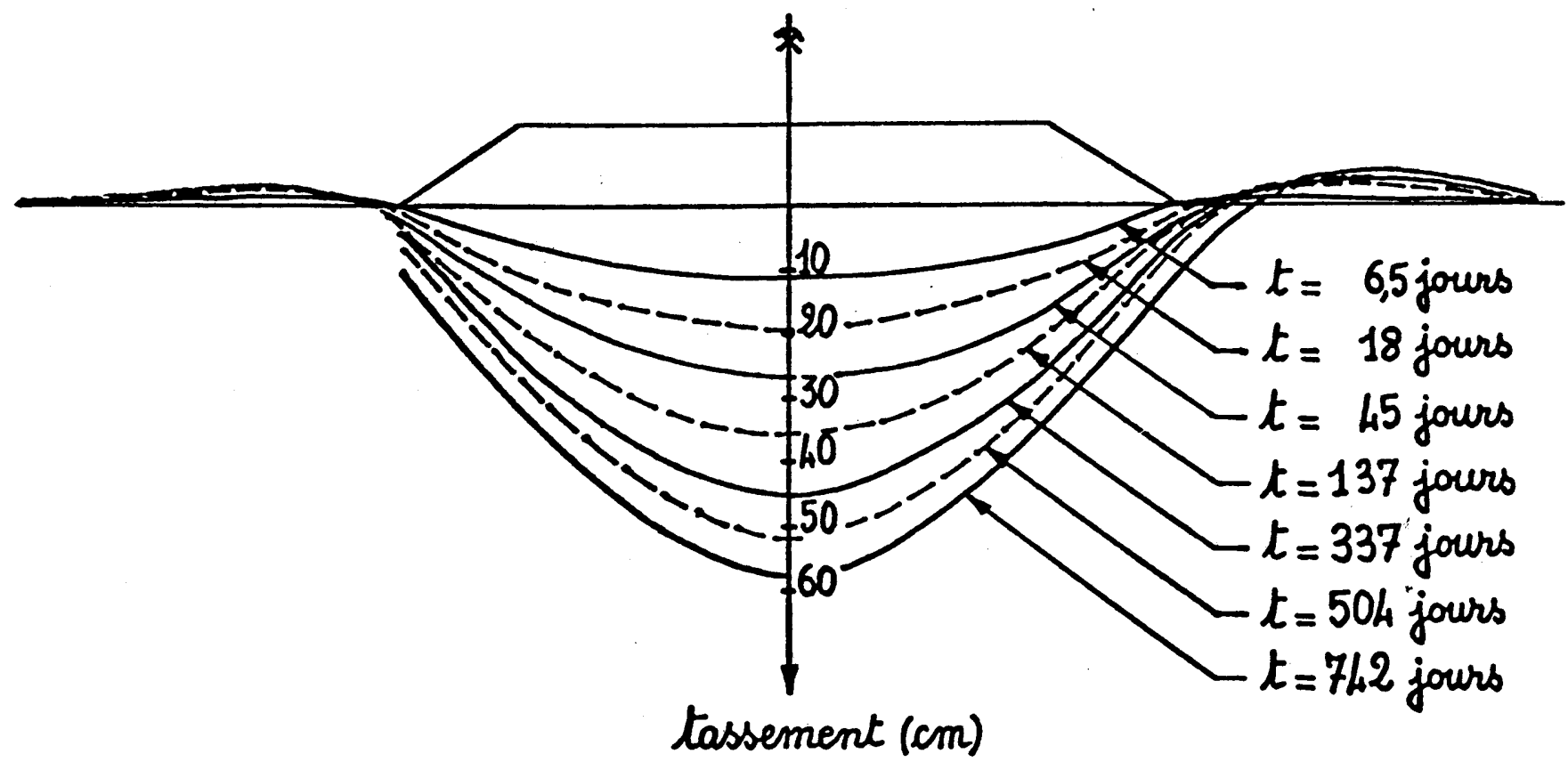

Fig. 10 Evolution des tassements dans le profil $\mathrm{k}$
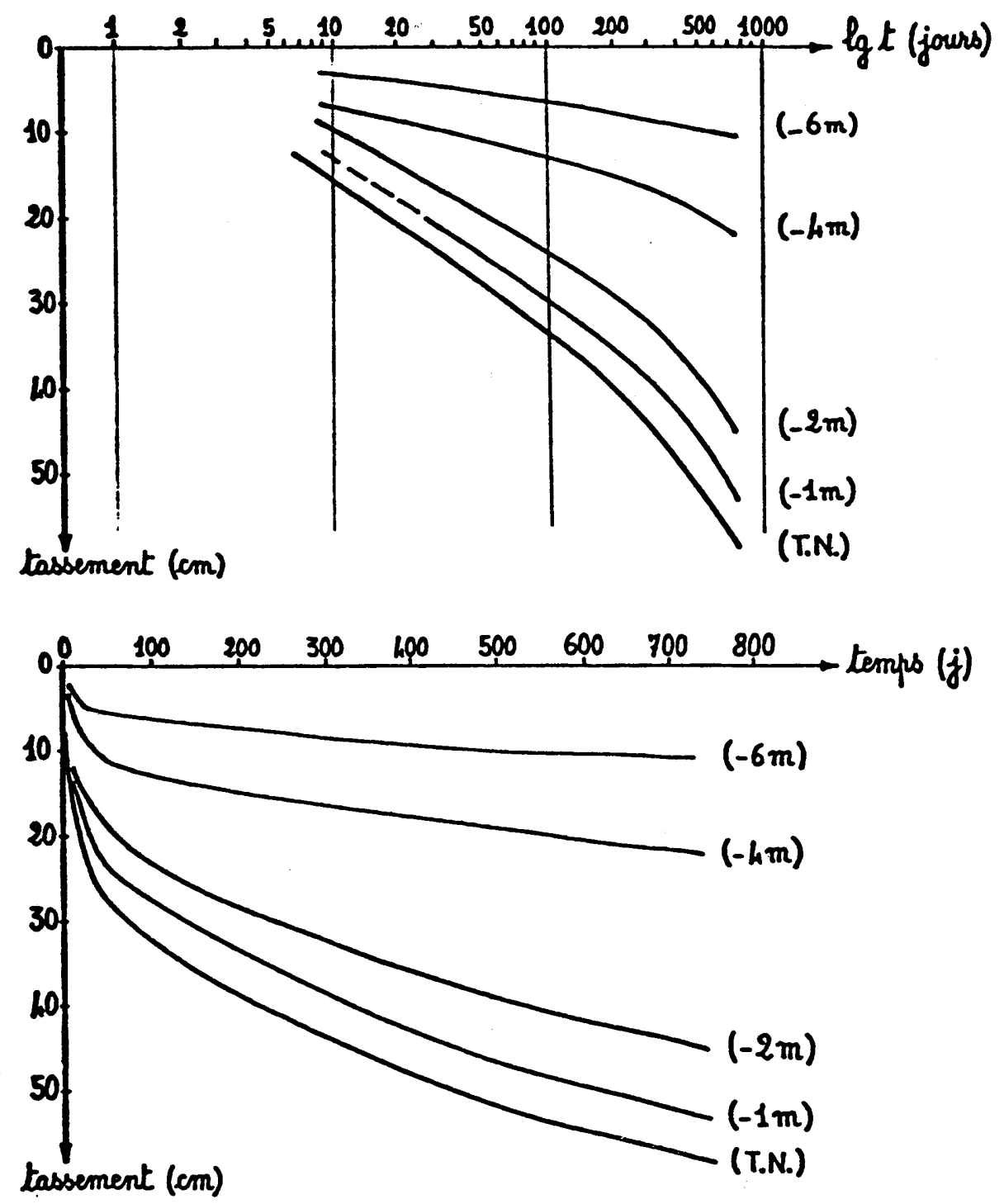

Fig. 11 Tassements du sol dans l'axe du remblai 


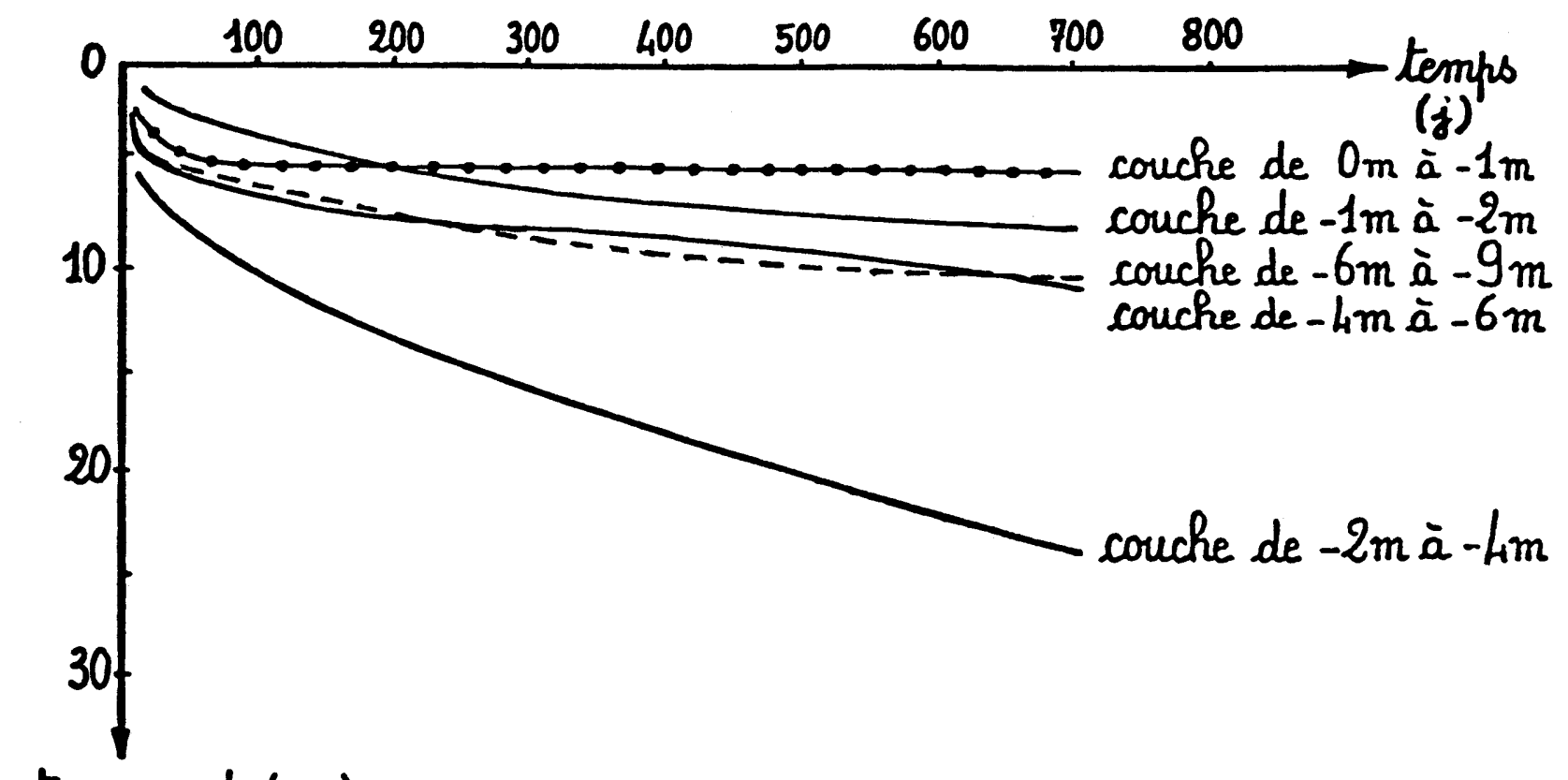

tassement (cm)

Fig. 12 Tassement du sol par couche

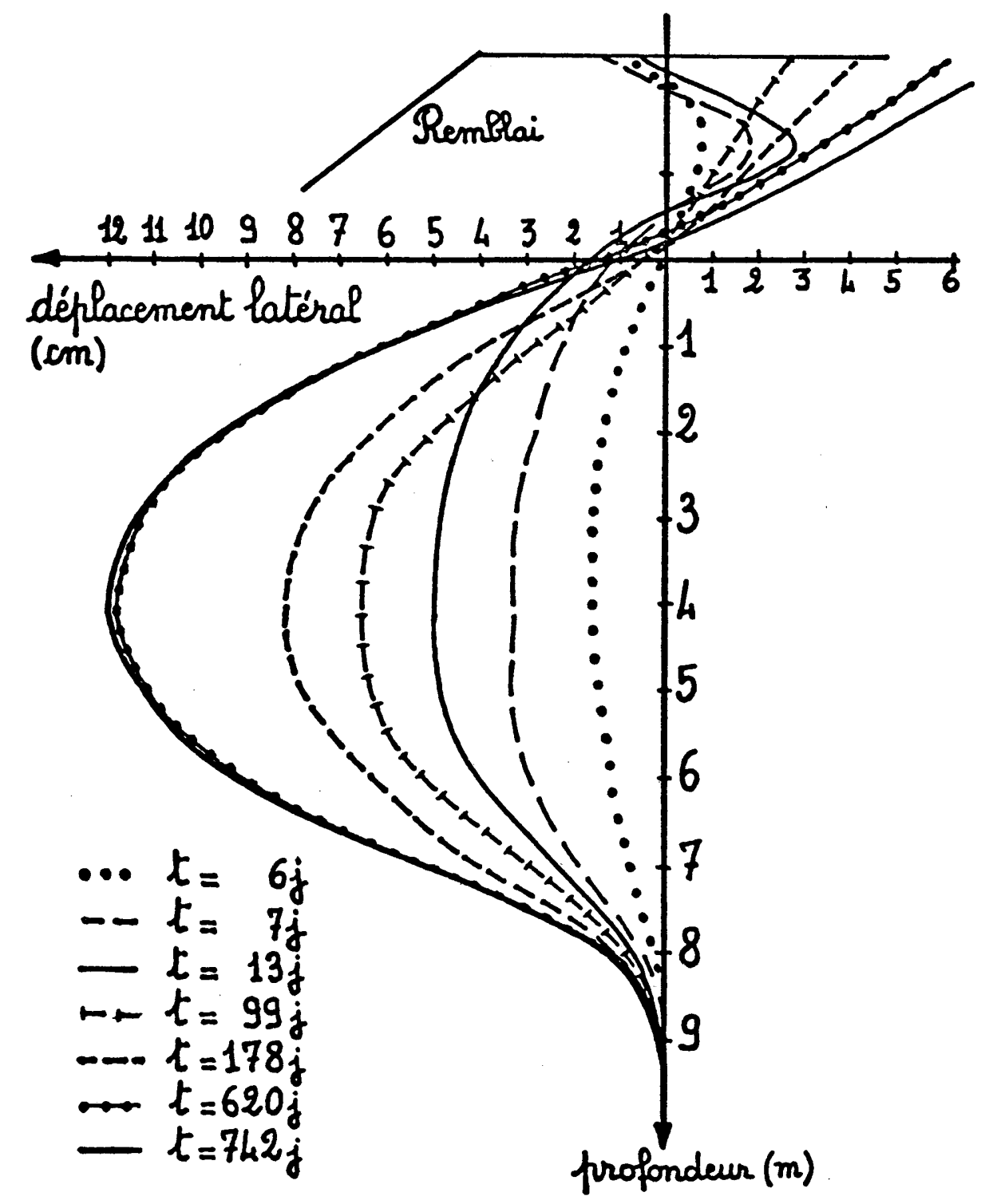

Fig. 13 Déplacements latéraux sous la crête du remblai 
noté un mouvement analogue du tube inclinométrique T71 (fig. 13) pendant cette période, qui a vu également un tassement important du pied du remblai. Cet ensemble de constatations montre que, contrairement d l'hypothèse couramment admise, il existe des déformations horizontales différées non négligeables dans le sol de fondation de ce remblai. Si la stabilisation de ces déformations se confirme, il restera néanmoins difficile de relier ces déformations à la consolidation, qui est loin d'être terminée dans la plus grande partie de la couche de sol compressible.

\subsection{Pressions interstitielles}

Les isochrones de pression interstitielle sous le remblai B
(" u mesuré » moins " $u$ hydrostatique moyen » pour une nappe à $-1 \mathrm{~m}$ ) traduisent le rôle non négligeable de la "dimension horizontale" du problème (fig. 14) : au cours du temps la zone des hautes pressions centrales tend à se rétrécir tandis que les isochrones de faible pression interstitielle ont tendance à s'étendre horizontalement sous les talus du remblai. Ces phénomènes sont typiques d'une consolidation bidimensionnelle.

On note également qu'en aucun point du sol de fondation la surpression interstitielle générée pendant la construction du remblai n'est égale à la charge appliquée, ce qui peut provenir de l'anisotropie mécanique ou de la nonsaturation du sol ou même d'une consolidation primaire très rapide.
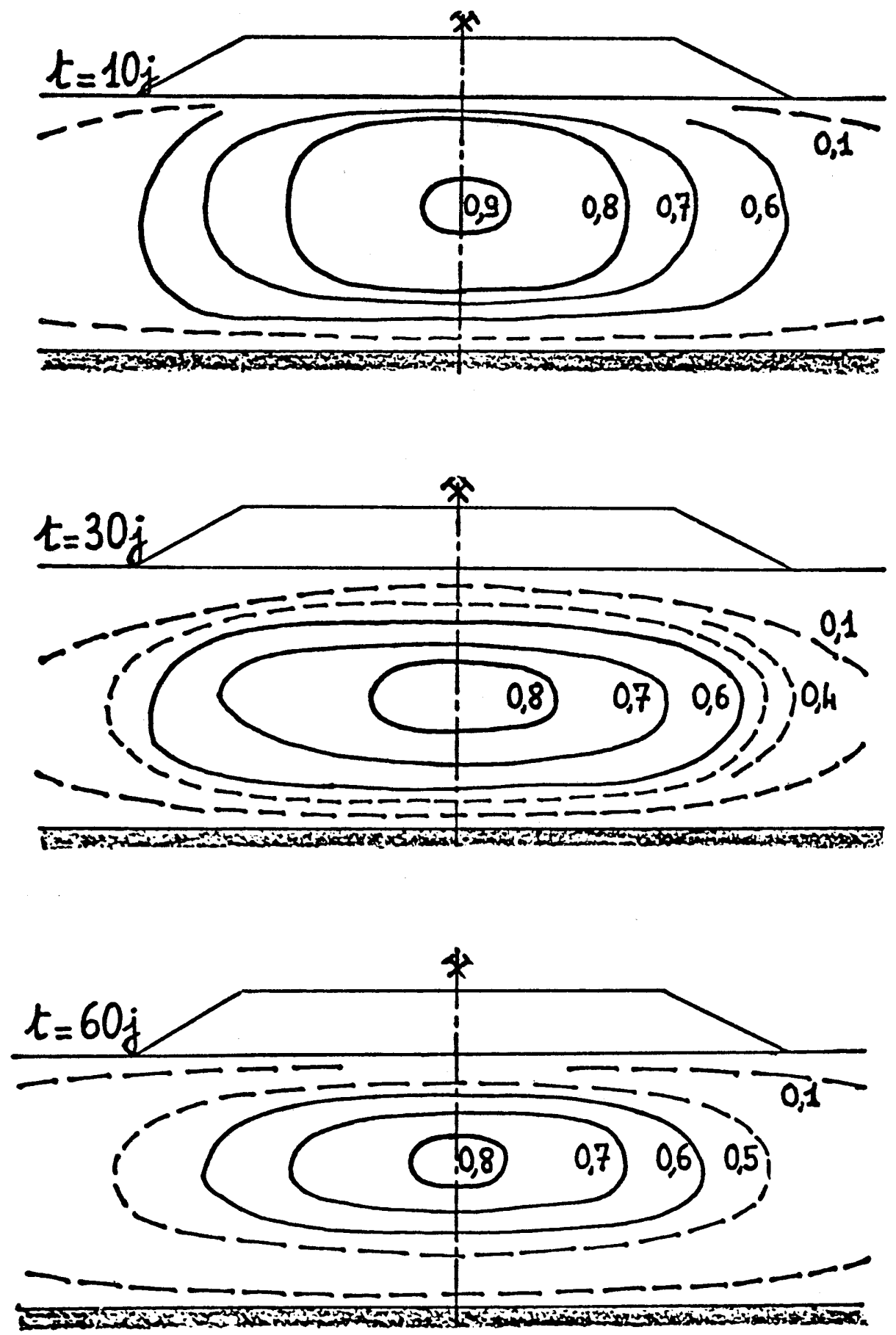

Fig. 14 Courbes d'égales valeurs de $r_{u}=\frac{\Delta u}{\gamma_{r} H_{r}}$ 


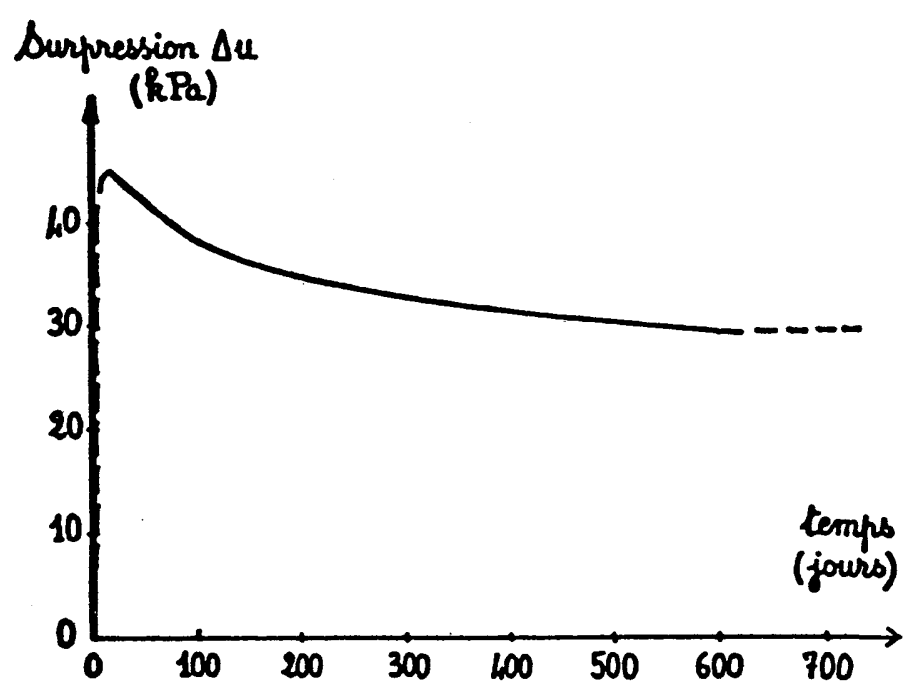

Fig. 15 Surpression interstitielle à mi-couche $(-5 \mathrm{~m})$

Deux ans après la construction du remblai, la surpression interstitielle en milieu de couche $(5 \mathrm{~m}$ de profondeur par rapport au terrain naturel) est encore élevée $(60 \%$ de la charge apportée par le remblai) et la consolidation de la couche d'argile est loin d'être terminée (fig. 15).

\subsection{Autres mesures}

Les capteurs de contraintes totales mis en place sous le remblai se sont avérés peu fiables au cours du temps (dérive des jauges de mesure) et les mesures effectuées sont pratiquement inexploitables, au point que l'on envisage de renoncer à ce type de mesure pour le remblai $C$ à construire sur le site de Cubzac-les-Ponts.

Les mesures de déformations horizontales relatives (rapprochement ou éloignement de points distants de $1 \mathrm{~m}$ ou de $50 \mathrm{~cm}$ ) ont permis de confirmer le comportement intuitif de la base du remblai: extension de la partie centrale et contraction du pied.

Le relevé topographique des jalons n'a pas soulevé de problème particulier.

\section{Prévisions des tassements et déformations latérales}

Les valeurs moyennes des principaux paramètres de compressibilité et de perméabilité pour chaque mètre de la couche compressible sont indiquées sur la figure 16 .

Les paramètres $A_{V}$ et $B_{v}$ sont ceux de la relation $e=A_{V}+B_{V} I g k v$. Les paramètres $A_{H}$ et $B_{H}$ sont ceux de la relation analogue pour la perméabilité horizontale. Tous les calculs ont été effectués sur la base de ces valeurs.

\subsection{Amplitudes des déformations} du sol de fondation

\subsubsection{Méthode œdométrique classique}

L'utilisation pour chaque couche de $1 \mathrm{~m}$ de la formule classique

$$
\frac{\Delta h}{h_{o}}=\frac{C s}{1+e_{o}} \lg \frac{\sigma^{\prime} p}{\sigma_{\text {VO }}^{\prime}}+\frac{C c}{1+e_{o}} \lg \frac{\sigma^{\prime} f}{\sigma^{\prime} p}
$$

avec les valeurs indiquées dans la figure 16 conduit à prédire un tassement final " œdométrique » de $0,79 \mathrm{~m}$.

\subsubsection{Méthode pressiométrique}

Les résultats des essais pressiométriques réalisés sur le site ont été utilisés pour calculer le tassement final par la méthode développée par Ménard (1965) et utilisée normalement pour les tassements des fondations (Fascicule Fond 72, 1972). Pour un sol stratifié cette méthode repose sur la formule semi-empirique :

$$
s=\sum_{i} \frac{\alpha_{i} \beta_{i} p_{i} h_{i}}{E_{i}}
$$

dans laquelle on utilise les notations suivantes :

$s$ - tassement de la surface du sol,

$\alpha$ - coefficient de structure, fonction de la nature du sol,

$\beta$-coefficient dépendant du coefficient de sécurité $F$

$$
\left(\frac{2}{3} \cdot \frac{F}{F-1} \text { si } F<3 \text { ou } 1 \text { si } F>3\right)
$$

$P_{i}$ - pression verticale créée par le remblai au centre de la ième couche,

\begin{tabular}{|c|c|c|c|c|c|c|c|c|c|c|c|c|c|c|c|c|c|c|}
\hline \multirow{3}{*}{ couches } & \multirow{3}{*}{$\therefore$} & \multirow{3}{*}{$\begin{array}{l}\sigma^{\circ} \\
k P a\end{array}$} & \multirow{3}{*}{ kpd } & \multirow{3}{*}{$c_{8}$} & \multirow{3}{*}{$c_{c}$} & \multicolumn{8}{|c|}{ Dralnage vertical } & \multicolumn{3}{|c|}{ Drainage horlrontal } & \multirow{3}{*}{$\begin{array}{l}\text { pour } \\
\sigma^{\circ}=\sigma^{\circ} \\
k h / k v\end{array}$} & \multirow{3}{*}{$c_{\alpha}$} \\
\hline & & & & & & \multirow{2}{*}{ A } & \multirow{2}{*}{$\mathbf{B}_{\mathbf{v}}$} & \multicolumn{2}{|c|}{$\sigma^{\circ}=\sigma_{0}^{\circ}$} & \multicolumn{2}{|c|}{$0^{\circ}=0_{0}^{\circ}+\frac{48,3}{2}$} & \multicolumn{2}{|c|}{$\sigma^{\circ}=0_{0}^{\circ}+48,3$} & \multirow{2}{*}{$A_{\mathrm{h}}$} & \multirow{2}{*}{$\mathrm{B}_{\mathrm{h}}$} & \multirow{2}{*}{$\frac{0^{-}=0_{0}^{-}}{0 k_{h}}$} & & \\
\hline & & & & & & & & $\begin{array}{l}k_{v} \\
c \pi / \sqrt{s}\end{array}$ & $\begin{array}{c}c . \mathrm{v} \\
\mathrm{cm}^{2} / \mathrm{s}\end{array}$ & $\begin{array}{l}k_{v} \\
o N / s\end{array}$ & $\begin{array}{c}c v \\
c m^{2} / s\end{array}$ & $\begin{array}{c}k_{v} \\
\mathrm{c} / \mathrm{s} / \mathrm{s}\end{array}$ & $\begin{array}{c}c \mathrm{v} \\
\mathrm{cm}^{2} / \mathrm{s}\end{array}$ & & & & & \\
\hline o a $1 \mathrm{~m}$ & 1 & 8,4 & 78 & 0,04 & 0,28 & 2,2 & 0,17 & $9.10^{-8}$ & $8,6 \cdot 10^{-4}$ & $6,5 \cdot 10^{-8}$ & $2,4 \cdot 10^{-3}$ & $5,7.10^{-8}$ & $3,6 \cdot 10^{-3}$ & 2,3 & 0,19 & $4,6 \cdot 10^{-8}$ & 0,51 & 0,15 \\
\hline 1 a $2 \mathrm{~m}$ & 2,6 & 20 & 68 & 0,05 & 1,22 & 8,24 & 0,81 & $1,25.10^{-7}$ & $4,2 \cdot 10^{-3}$ & $1,2 \cdot 10^{-7}$ & $8,7.10^{-3}$ & $1,15.10^{-7}$ & $1,3.10^{-2}$ & & & & & 0,05 \\
\hline $2 \mathrm{a} 3 \mathrm{~m}$ & 3,15 & 24 & 36 & 0,21 & 1,75 & 13,4 & 1,39 & $4,3.10^{-8}$ & $4,7.10^{-4}$ & $2,5 \cdot 10^{-8}$ & $6,6 \cdot 10^{-5}$ & $1,7.10^{-8}$ & $5,7.10^{-5}$ & 11 & 1,13 & $1,4 \cdot 10^{-7}$ & 3,26 & 0,08 \\
\hline $3 \times 4 \mathrm{~m}$ & 3,3 & 28 & 36 & $|0,18|$ & 1,70 & 10,5 & 1,03 & $1 \quad .10^{-7}$ & $1,6 \cdot 10^{-3}$ & $5,4 \cdot 10^{-8}$ & $1,5.10^{-4}$ & $2,9.10^{-8}$ & $1,1.10^{-4}$ & 12 & 1,24 & $3,6 \cdot 10^{-7}$ & 3,60 & 0,043 \\
\hline $415 \mathrm{~m}$ & 2,42 & 32 & 38,5 & 0,12 & 1,28 & 8,95 & 0,93 & $9,7 \cdot 10^{-8}$ & $2 \cdot 10^{-3}$ & $5,6 \cdot 10^{-8}$ & $1,8 \cdot 10^{-4}$ & $3,4 \cdot 10^{-8}$ & $1,5 \cdot 10^{-4}$ & 10 & 1,07 & 1. $.10^{-7}$. & 3,09 & 0,034 \\
\hline 5 a $6 \mathrm{~m}$ & 2,07 & 36 & 41 & 0,10 & 1,18 & 7,46 & 0,78 & $1,2 \cdot 10^{-7}$ & $3,2 \cdot 10^{-3}$ & $6,8 \cdot 10^{-8}$ & $2,3.10^{-4}$ & $4,1.10^{-8}$ & $1,8 \cdot 10^{-4}$ & 6,23 & 0,65 & $1 ; 9.10^{-7}$ & 2,42 & 0.030 \\
\hline $6 \mathrm{a} 7 \mathrm{~m}$ & 2,13 & 40 & 46 & 0,08 & 1,11 & 7,01 & 0,70 & $1,1.10^{-7}$ & $3,9.10^{-3}$ & $6,4 \cdot 10^{-8}$ & $2,5 \cdot 10^{-4}$ & $3,9 \cdot 10^{-8}$ & $2 \cdot 10^{-4}$ & 8 & 0,84 & $1,1.10^{-7}$ & 1 & 0,023 \\
\hline $7 \mathrm{a} 8 \mathrm{~m}$ & 2,5 & 45 & 57 & 0,10 & 1,30 & 8,42 & 0,84 & $9.10^{-8}$ & $3,3.10^{-3}$ & $6,6 \cdot 10^{-8}$ & $2,7.10^{-4}$ & $4,1 \cdot 10^{-8}$ & $2,2 \cdot 10^{-4}$ & $p, 4$ & 0,74 & $1,5.10^{-7}$ & 1,67 & 0,039 \\
\hline $8 \mathrm{a} 9 \mathrm{~m}$ & 2,2 & 48 & 68 & 0,11 & 1,20 & 7,52 & 0,75 & $8,2 \cdot 10^{-8}$ & $2,6.10^{-3}$ & $7.10^{-8}$ & $3 \cdot .10^{-4}$ & $4,4 \cdot 10^{-8}$ & $2,4 \cdot 10^{-4}$ & & & & & 0,035 \\
\hline
\end{tabular}

$\mathrm{H}_{\mathrm{i}}$ - épaisseur de la ième touche de sol,

$E_{i}$ - module pressiométrique dans la ième couche de sol.

Fig. 16 Synthèse des essais de compressibilité en laboratoire 
Le calcul est assez sensible aux valeurs choisies pour le coefficient $\alpha$. En supposant qu'à toute profondeur la pression $P$ est égale au poids du remblai, on obtient, pour les valeurs de $E$ indiquées dans la figure 16 :

$S=79 \mathrm{~cm}$ si $\alpha=1$ dans la croûte surconsolidée de surface et $\alpha=0,5$ dans le reste du sol,

$\mathrm{S}=68 \mathrm{~cm}$ si $\alpha=2 / 3$ dans la croûte de surface et $\alpha=0,9$ dans le reste du sol.

(Le calcul est effectué sur les 13 premiers mètres du sol, c'est-à-dire que l'on tient compte des 4 premiers mètres du substratum graveleux, pour lesquels on dispose des caractéristiques pressiométriques).

\subsection{3 Élasticité linéaire (tassement à court terme)}

Le tassement instantané du sol peut se calculer à partir des valeurs moyennes des paramètres $\nu$ et $E$ déterminées en laboratoire dans des essais triaxiaux non consolidés non drainés, à des niveaux de contraintes voisins de ceux existant en place sous le remblai.

Les abaques de Giroud (Tome 2 ; 1973) donnent

$\mathrm{S}=0,215 \mathrm{~m}$ pour $\nu=0,4$ et $\mathrm{E}=1220 \mathrm{kPa}$.

$\mathrm{S}=0,076 \mathrm{~m}$ pour $\nu=0,5$ et $\mathrm{E}=1800 \mathrm{kPa}$.

(Les valeurs de $E$ ont été obtenues selon la méthode approchée rappelée par Bourges (1973), en évaluant les contraintes élastiques par les abaques de Poulos).

On a aussi effectué un calcul élastique bidimensionnel sur un modèle de sol bicouche (programme Birug du L.C.P.C. Mandagaran, 1973), avec pour paramètres d'élasticité :

$$
\begin{aligned}
& \text { de } 0 \quad a ̀-1,5 \mathrm{~m} \mathrm{E}=4000 \mathrm{kPa} . \\
& \text { de }-1,5 \mathrm{~m} \text { à }-9 \mathrm{~m} \mathrm{E}=1600 \mathrm{kPa} .
\end{aligned}
$$

Ce calcul a été réalisé pour deux valeurs du coefficient de Poisson $v$, supposé égal dans les deux couches. Le tassement à court terme du centre du remblai vaut

$$
\begin{array}{ll}
\text { pour } \nu=0,4 & S=0,142 \mathrm{~m} . \\
\text { pour } \nu=0,48 & \mathrm{~S}=0,083 \mathrm{~m} .
\end{array}
$$

\subsection{4 Élasticité linéaire (tassement final)}

Le calcul en élasticité du tassement final du remblai peut être effectué à l'aide de paramètres $\mathrm{E}^{\prime}$ et $\nu^{\prime}$ déduits d'essais triaxiaux drainés ou d'essais de compressibilité à l'œdomètre. La valeur moyenne de $\mathrm{E}^{\prime}$ déduite des essais œdométriques dans I'hypothèse où $\nu^{\prime}=0,3$ vaut $E^{\prime} M=300 \mathrm{kPa}$. Les abaques de Giroud (1973) donnent dans ce cas $\left(E^{\prime}=300 \mathrm{kPa}, \nu^{\prime}=0,3\right)$ :

$$
\mathrm{S}=1,14 \mathrm{~m} \text {. }
$$

On pourrait penser à utiliser la formule indiquée par Giroud et Cordary (1976) pour déduire des paramètres d'élasticité à court terme $\left(E_{U}, \nu \cup=0,5\right)$ la valeur des paramètres d'élasticité à long terme $\left(E^{\prime}, v^{\prime}=0,3\right)$ :

$$
E^{\prime}=E_{U}\left[1-A B\left(1-\nu^{\prime}\right)\right] \text {, }
$$

$A$ et $B$ désignant les coefficients de pression interstitielle de Skempton. La valeur des coefficients $A$ et $B$ est malheureusement difficile à déterminer en pratique. Pour $A B\left(1-\nu^{\prime}\right)=0,5$ on trouverait $E^{\prime}=900 ! k P a$, d'où $\mathrm{S}=0,38 \mathrm{~m}$. II faudrait que $A B=1,2$ pour que l'on retrouve $E^{\prime}=300 \mathrm{kPa}$. D'autre part, la formule établie sous certaines hypothèses dans le cadre de l'élasticité linéaire isotrope,

$$
\frac{E}{1+V}=\frac{E^{\prime}}{1+V^{\prime}}
$$

conduit pour $\nu^{\prime}=0,3$ a $S=0,30$ m et $S=0,22 \mathrm{~m}$ respectivement pour les deux hypothèses de calcul du paragraphe précédent, ce qui confirme la conclusion de Giroud et Cordary (1976) sur le peu de valeur de cette formule.

\subsubsection{Modèle Cam-Clay modifié}

Le remblai B de Cubzac a fait l'objet d'un calcul par éléments finis utilisant le modèle Cam-Clay modifié développé par le Groupe de Mécanique des Sols de l'Université de Cambridge. Ce calcul a permis d'estimer les valeurs finales des déformations du sol de fondation du remblai (Dang et Magnan, 1977). Ces résultats sont comparés aux mesures sur la figure 18. Le tassement calculé du centre du remblai valait $0,83 \mathrm{~m}$.

\subsection{Vitesses de déformation} du sol de fondation

\subsubsection{Calcul unidimensionnel avec l'abaque de Terzaghi}

La théorie classique de la consolidation unidimensionnelle de Terzaghi, appliquée dans l'hypothèse d'une couche de $9 \mathrm{~m}$ drainée des deux côtés et de coefficient de consolidation

$$
\mathrm{C}_{v}=2.10^{-8} \mathrm{~m}^{2} / \mathrm{S} \text {. }
$$

(valeur moyenne des $C_{V}$ mesurés à l'œdomètre entre 3 et $9 \mathrm{~m}$ de profondeur sous une contrainte effective égale à $\sigma_{v}^{\prime}=\sigma_{v o}^{\prime}+\frac{\Delta \sigma_{v}}{2}$ ), conduit aux résultats présentés sur la figure 17 : courbe de tassement de la surface du sol au cours du temps et courbe de dissipation de la surpression interstitielle moyenne.

Les tassements ont été obtenus en multipliant le tassement final calculé en 4.1.1 par le degré de consolidation $U$ de la théorie de Terzaghi.

Ce degré de consolidation a été calculé pour deux distributions initiales de la surpression interstitielle :

- une distribution initiale uniforme $\Delta u(t=0)=\Delta \sigma$,

- une distribution initiale sinusoïdale.

Les courbes correspondant à la distribution uniforme sont affectées de l'indice "1u», les courbes correspondant à la distribution sinusoïdale sont désignées par «1s ».

\subsubsection{Calcul unidimensionnel} (programme Conmult, Magnan et al, 1978)

Le programme Conmult du L.C.P.C. permet la prise en compte des variations des paramètres au cours de la consolidation ainsi que du fluage du sol de fondation, décrit selon le modèle de Bjerrum (1972).

II a été utilisé avec les données de la figure 16 pour calculer la consolidation unidimensionnelle du sol sans fluage puis avec fluage. Les courbes de consolidation correspondantes sont tracées sur la figure 17. On y distingue :

- les courbes obtenues en l'absence de fluage pour la distribution observée des surpressions interstitielles à la fin de la construction du remblai, pour une nappe au niveau du terrain naturel (2so) et pour une nappe à $1 \mathrm{~m}$ de profondeur (2s1),

- les courbes obtenues en tenant compte du fluage pour la distribution observée des surpressions interstitielles à la fin de la construction du remblai (2f).

\subsubsection{Dissipation bidimensionnelle} des pressions interstitielles

Les essais de laboratoire ont mis en évidence une anisotropie de perméabilité non négligeable $\frac{\mathrm{kh}}{\mathrm{kv}} \simeq 2,5$. Pour tenir compte de cette anisotropie, on a mis au point un programme de calcul sur ordinateur permettant de simuler l'écoulement bidimensionnel de l'eau dans le sol dans I'hypothèse où la déformation du squelette du sol reste verticale (modèle œdométrique).

Les courbes (3) de la figure 17 traduisent les résultats de ce calcul effectué en supposant que la distribution initiale des pressions interstitielles sous le remblai est sinusoïdale en fonction de la profondeur et que le maximum de cette sinusoïde est égal au poids du remblai à la verticale du profil considéré (le choix de la condition initiale sous les bords du remblai n'a pas grande influence sur le résultat des calculs au-delà des premiers jours de la consolidation).

4.2.4 Calcul du tassement œdométrique à partir des pressions interstitielles mesurées

Les isochrones des surpressions interstitielles sous le 


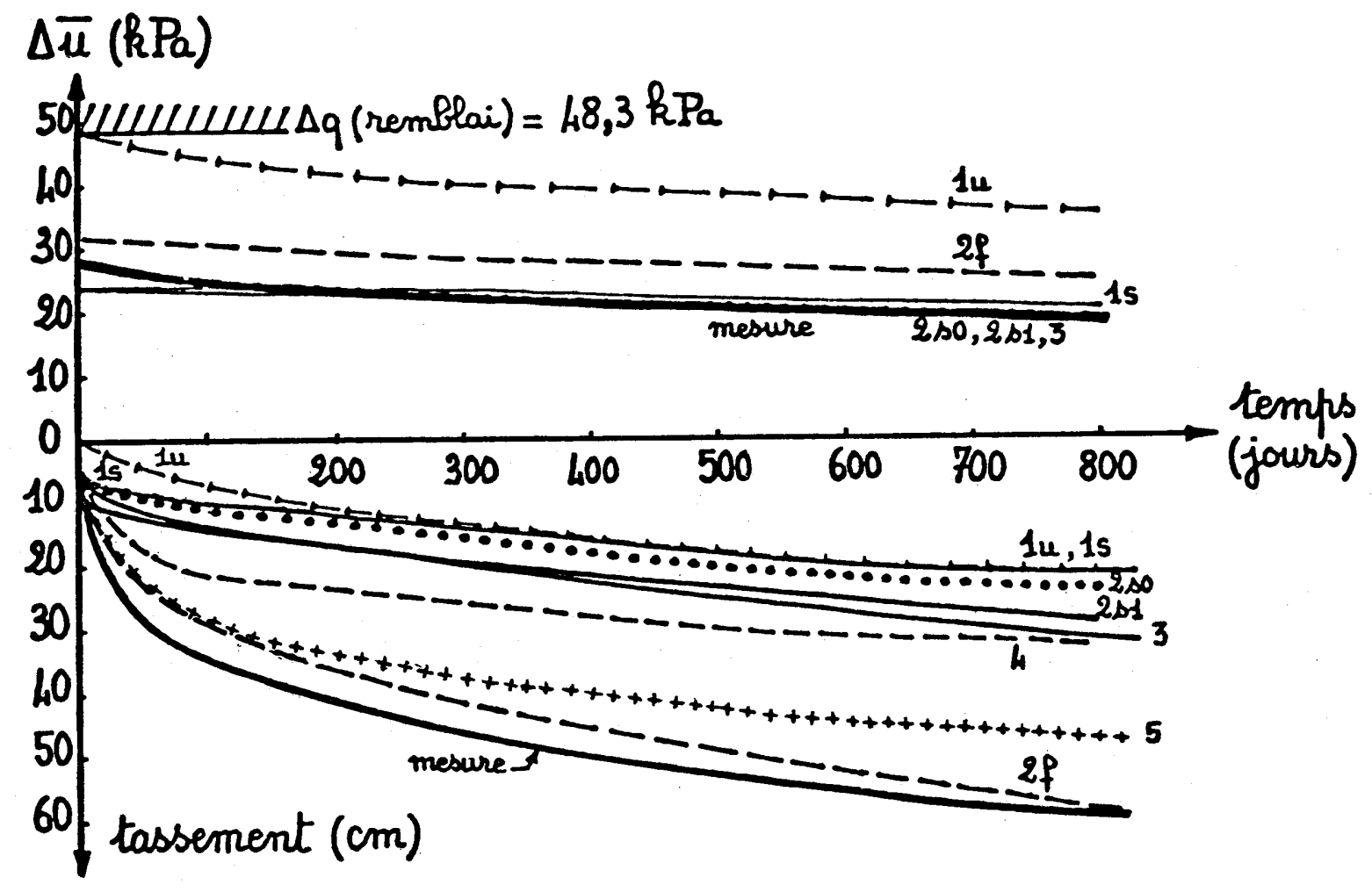

Fig. 17 Comparaison des calculs de consolidation et des mesures

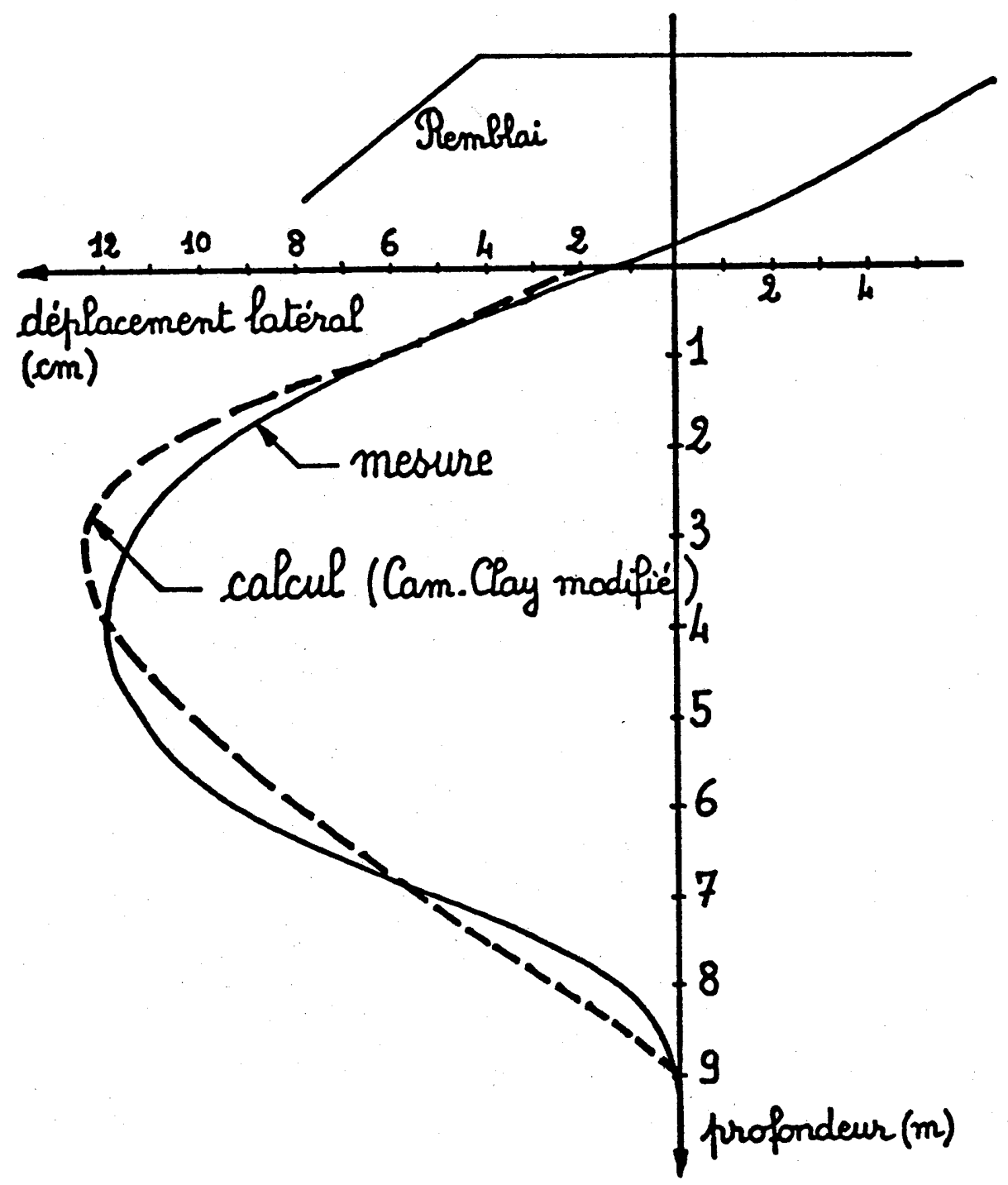

Fig. 18 Comparaison des déplacements latéraux mesurés et calculés (tube inclinométrique IL7, situé en crête du talus du remblai) 
centre du remblai sont suffisamment bien connues pour que l'on puisse calculer le tassement de chaque mètre du sol de fondation à l'aide de la formule

$$
\frac{\Delta h_{i}(t)}{h_{o i}}=\frac{C_{s i}}{1+e_{o i}} \lg \frac{\sigma_{p i}^{\prime}}{\sigma_{o i}^{\prime}}+\frac{C_{c i}}{1+e_{o i}} \lg \frac{\sigma_{i}^{\prime}(t)}{\sigma_{p i}^{\prime}}
$$

les indices. $i$ indiquant que les valeurs des paramètres sont celles de la ième couche du sol. La contrainte effective $\sigma_{i}^{\prime}(t)$ se déduit de la pression interstitielle en milieu de couche $U_{j}(t)$ et de la surcharge due au poids du remblai $\Delta \sigma$ à l'aide de la formule

$$
\sigma_{i}^{\prime}(t)=\sigma^{\prime}{ }_{\text {voi }}+\left[\Delta \sigma-U_{i}(t)\right]
$$

La courbe de tassement obtenue est la courbe (4) de la figure 17. Le tassement final du centre du remblai estimé par cette méthode est de 0,91 m.

\subsubsection{Prise en compte du fluage (compression secondaire) par la méthode de Gibson \& Lo}

Du fait de la forte épaisseur de la couche compressible, le paramètre $\mathrm{N}$ de la méthode de Gibson et Lo (1961) vaut près de 3000 , ce qui rend négligeable l'influence du fluage sur le déroulement de la consolidation. L'amplitude du tassement est par contre augmentée d'environ $20 \%$. La courbe correspondante n'a pas été représentée sur la figure 17, où elle aurait été comprise entre les courbes 2 so et 2 s1.

\subsubsection{Prise en compte du fluage}

par la méthode de Buisman et Koppejan

La méthode classique de Buisman (1936) et Koppejan (1948) permet d'évaluer le tassement de fluage du sol à l'aide d'un terme $\frac{C_{\alpha} h_{O}}{1+e_{0}} \lg t$ ajouté au tassement final de consolidation primaire (t exprimé en jours).

Si l'on ajoute au tassement calculé sans fluage par la méthode de Terzaghi (courbe $1 \mathrm{~s}$ ) le terme 0,09 lgt correspondant aux valeurs moyennes de $\mathrm{C} \alpha$ et eo dans la couche compressible, on obtient la courbe (5) de la figure 17.

La contribution du fluage au tassement total du centre du remblai peut être évaluée à $S_{F L}=0,29 \mathrm{~m}$ pour $\mathrm{t}=5$ ans, ce qui conduit à un tassement final voisin de $1,08 \mathrm{~m}$ par cette méthode de type œdométrique.

\section{Discussion des résultats}

\subsection{Amplitude des tassements}

On considère généralement comme satisfaisante la prévision du tassement du centre des remblais par la méthode œdométrique sous réserve que l'épaisseur de la couche compressible soit au plus de l'ordre de la largeur du remblai. Les éléments disponibles à l'heure actuelle sur le remblai $B$ du site expérimental de Cubzac-les-Ponts ne permettent pas de tirer des conclusions définitives, mais il semble que :

- le tassement œdométrique calculé sans tenir compte du fluage sera sensiblement inférieur au tassement réel;

- le tassement œdométrique calculé en tenant compte d'une loi de fluage logarithmique sera un peu supérieur au tassement réel;

- le tassement évalué en élasticité linéaire avec des valeurs moyennes $\mathrm{E}^{\prime}$ et $\nu^{\prime}$ déduites des essais œdométriques sera trop important, bien qu'il ne tienne pas compte du fluage du sol;

- le tassement déduit des formules pressiométriques sera trop faible ;

- le tassement calculé sur la base du Modèle "Cam-Clay modifié" sera également un peu trop faible.

Ces conclusions devront être confirmées dans quelques années quand le tassement du remblai se sera stabilisé.
II faut noter toutefois que, si les valeurs des tassements "œdométriques » sont bien définies, les autres calculs résultent d'une appréciation un peu subjective de la valeur de $E^{\prime}$ dans la couche compressible, du coefficient $\alpha$ à utiliser dans la formule pressiométrique et de plusieurs paramètres du modèle Cam-Clay modifié et que les tassements estimés par ces méthodes représentent autant le schéma de calcul que le choix de paramètres utilisés.

\subsection{Déplacements latéraux}

Le seul calcul réalisé sur ce point (Modèle Cam-Clay modifié) est prometteur puisque les déformées réelle et théorique étaient presque confondues au bout de deux ans (Fig. 18). Mais l'évolution ultérieure des déplacements latéraux permettra seule de juger la validité de ce modèle de calcul, par ailleurs sensible, comme on l'a déjà indiqué, au choix des paramètres.

\subsection{Vitesses de tassement}

Les courbes représentées sur la figure 17 montrent que :

- la prise en compte du fluage du sol permet seule d'approcher la courbe de tassement réel du centre du remblai :

- pour les tassements, la théorie de Terzaghi appliquée de façon élémentaire $S(t)=S_{\infty}$. $U(t)$ donne des résultats pratiquement équivalents aux méthodes de calcul plus compliquées tenant compte d'un comportement plus complexe du sol (programme Conmult), mais la prévision des pressions interstitielles est peu fiable par cette théorie ;

- la prise en compte simultanée du fluage du sol, des variations de la perméabilité au cours de la consolidation et de la non-linéarité de la loi de compressibilité unidimensionnelle permet de calculer un tassement très voisin du tassement observé. Ce résultat demande toutefois à être confirmé par des études complémentaires sur d'autres remblais réels.

\section{Conclusion}

Le remblai $B$ du site expérimental des Laboratoires des Ponts et Chaussées à Cubzac-les-Ponts (Gironde) fait l'objet d'observations régulières depuis sa construction en 1975. L'évolution observée des tassements, des déplacements latéraux et des pressions interstitielles au cours du temps constitue une bonne base pour la comparaison et l'étalonnage des méthodes de calcul disponibles pour la prévision du comportement des remblais courants de ce type ( $F=1,5$; couche de sol compressible peu épaisse). Sous réserve de l'évolution ultérieure du comportement du remblai, les comparaisons déjà réalisées montrent le rôle très important du fluage dans les déformations du sol moyennement organique du site expérimental et confirment les promesses de certaines des méthodes numériques développées ces dernières années pour l'étude du comportement au cours du temps des remblais sur sols mous.

\section{Remerciements}

La réalisation d'un chantier expérimental, son suivi pendant plusieurs années, les essais en place et en laboratoire et les calculs manuels et sur odinateurs nécessitent le travail persévérant mais obscur d'une équipe d'ingénieurs, de techniciens supérieurs, de techniciens et de stagiaires. Les trois auteurs reconnaissent ici leur rôle déterminant dans la réalisation de cette étude. 


\section{Références Bibliographiques}

BJERRUM L. - Rapport général sur le thème Embankments on soft ground, ASCE Specialty Conference on Performance of earth and earth-supported structures, PURDUE Univ., La Fayette, Indiana, 1972, Vol. 3, pp. 111-159.

BLONDEAU F., MIEUSSENS C., QUEYROI D., LEVILLAIN J.-P. PEIGNAUD M., VOGIEN M. - Instrumentation du remblai expérimental i $A$ ע de Cubzac-les-Ponts. International Symposium on Soft Clay, Bangkok, Thaïlande, juillet 1977, pp. 419-435.

BOURGES F., PAUTE J.-L. - Remblais sur sols compressibles : Méthodes d'étude utilisées pour les remblais expérimentaux. Bul. de Liaison des LPC, France, $\mathrm{n}^{\circ}$ Spécial T, mai 1973, pp. 60-87.

BUISMAN A. S. K. - Results of long duration settlement tests.

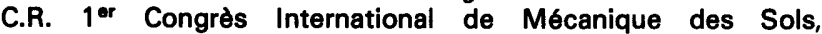
Cambridge, 1936, Mass., U.S.A., Vol. 1, pp. 103-105.

DANG M. T., MAGNAN J.-P.-Application des modeles élastoplastiques de l'Université de Cambridge au calcul du comportement d'un remblai sur sols mous (site expérimental de Cubzac-les-Ponts). Laboratoires des Ponts et Chaussées, France, Rapport de Recherche $n^{\circ} 74$, décembre 1977.

Fascicule FOND 72. - Ministère de l'Équipement, SETRA et LCPC, 1972.

GIBSON R. E., LO K. Y. -A theory of consolidation for soil exhibiting secondary compression. Norwegian Geotechnical Institute, Publication $n^{\circ} 41$, Oslo, 1961.
GIROUD J.-P. - Tables pour le calcul des fondations. Tome II, Dunod, Paris, 1973.

GIROUD J.-P., CORDARY D. - Prévision du tassement initial et du tassement différé des fondations superficielles. Annales de I'ITBTP, $n^{\circ} 335$, Série Sols-Fondations 127, janvier 1976, pp. 105-135.

KOPPEJAN A. W. - A formula combining the Terzaghi load compression relationship and the Buisman secular time effet. C.R. $2^{\circ}$ Congrès International de Mécanique des Sols, Rotterdam, 1948, pp. 32-37.

MAGNAN J.-P., BAGHERY S., BRUCY M., TAVENAS F. - Etude numérique de la consolidation unidimensionnelle avec prise en compte des variations de la perméabilité et de la compressibilité du sol, du fluage et de la non saturation. A paraitre' (1978).

MANDAGARAN B.-Calcul des contraintes dans un massif d'épaisseur limitée soumis a une charge trapézoidale. Laboratoires des Ponts et Chaussées, France, Rapport de Recherche $n^{\circ} 29$, novembre 1973.

MÉNARD L. - Regles pour le calcul de la force portante et du tassement des fondations en fonction des résultats pressiométriques. C.R. du $6^{\circ}$ Congrès International de Mécanique des Sols et Travaux de Fondation, Montréal, 1965, Vol. II, pp. 295-299.

VOGIEN M. - Étude du comportement avant la rupture d'un remblai expérimental construit sur sol mou à Cubzac-les-Ponts. Thèse de Docteur-Ingénieur, Université Pierre et Marie Curie, Paris. 